\title{
Bacterial biofilms, antibiotic resistance and healthcare-associated infections: a dangerous connection.
}

\author{
Bandeira, M.****, Carvalho, P.A.*, Duarte, A. ** and Jordao, L.*** \\ *Instituto Superior Técnico, Departamento de Bioengenharia, ICEMS, Av. Rovisco Pais, \\ 1049-001 Lisboa, PORTUGAL. \\ **Faculdade de Farmácia da Universidade de Lisboa, Departamento de Microbiologia e \\ Imunologia, iMed.UL, Av. Prof. Gama Pinto, 1649-003 Lisboa, PORTUGAL. \\ ***Instituto Nacional de Saude Dr Ricardo Jorge, Departamento de Doenças Infeciosas, Av. \\ Padre Cruz, 1649-016 Lisboa, PORTUGAL. \\ Email : maria.jordao@insa.min-saude.pt
}

In 2012, were estimated 6.7 million cases of healthcare-associated infections (HAI) either in long-term care facilities or acute-care hospitals from which result 37,000 deaths configuring a serious public health problem [1].

The etiological agents are diverse and often resistant to antimicrobial drugs. One of the mechanisms responsible for the emergence of drug resistance is biofilm assembly. Biofilms are defined as thin layers of microorganisms adhering to the surface of a structure, which may be organic or inorganic, together with the polymers that they secrete [2]. They are dynamic structures which experience different stages of organization with the ageing and are linked to an increase in bacterial resistance to host defense mechanisms, antibiotics, sterilization procedures other than autoclaving, persistence in water distribution systems and other surfaces. The understanding of bacteria organization within the biofilm and the identification of differences between planktonic and sessile forms of bacteria will be a step forward to fight HAIs.

Three bacterial isolates (K. pneumoniae 703;O:1, K. pneumoniae 45 and $K$. pneumoniae2948) were grown on Muller-Hinton plates or broth. Antibiotic susceptibility was evaluated by broth microdilution method and interpreted according to NCCLS guidelines. A similar assay was performed to evaluate biofilm susceptibility to antibiotics. Bacteria ability to assemble biofilms was assayed by the microtiter-plate test [3] being tested in both abiotic (materials present in healthcare units) and biotic (Hella cells) surfaces. The biofilm structure was assessed by scanning electron microscopy (SEM).

The ability to assemble biofilms was distinct, being $K$. pneumoniae 703;O:1 ranked as the best biofilm assembler and $K$. pneumoniae 2948 the worse. The SEM analysis of sessile (Figs.1A-1A.1) and planktonic (Fig.1B) forms of the same bacteria allowed the identification of structural differences which may be involved in virulence (Fig. 1). Bacteria ability to assemble biofilms seems to be independent of the abiotic structure (Fig.2). The same was not observed in biotic surfaces were K. pneumoniae 703;O:1 did not form a biofilm. This fact suggests that biofilm assembly in vivo is dependent of bacteria tropism. The minimum inhibitory concentration (MIC) determine for bacteria organized in biofilms is higher than for their planktonic forms. The increase ranges from 2 to 200 folds and is proportional to the ability of bacteria to assemble biofilms. Further studies will be conducted in order to prevent biofilm assembly within healthcare units which will result in a decrease of HAI and emergence of antibiotic resistant bacteria.

The authors acknowledge Fundação para a Ciência e Tecnologia for the grants C2008-C2008_P2 and PestOE/CTM/UI0084/2011.

[1] ECDC Europe; (2012), Annual epidemiological report, 2011.

[2] Bryers, J.D., Biotecthnol. Bioeng, 100, 1-18, 2008.

[3] Stepanovic S. et al., J Microbiol Methods, 40, 175-179, 2000. 


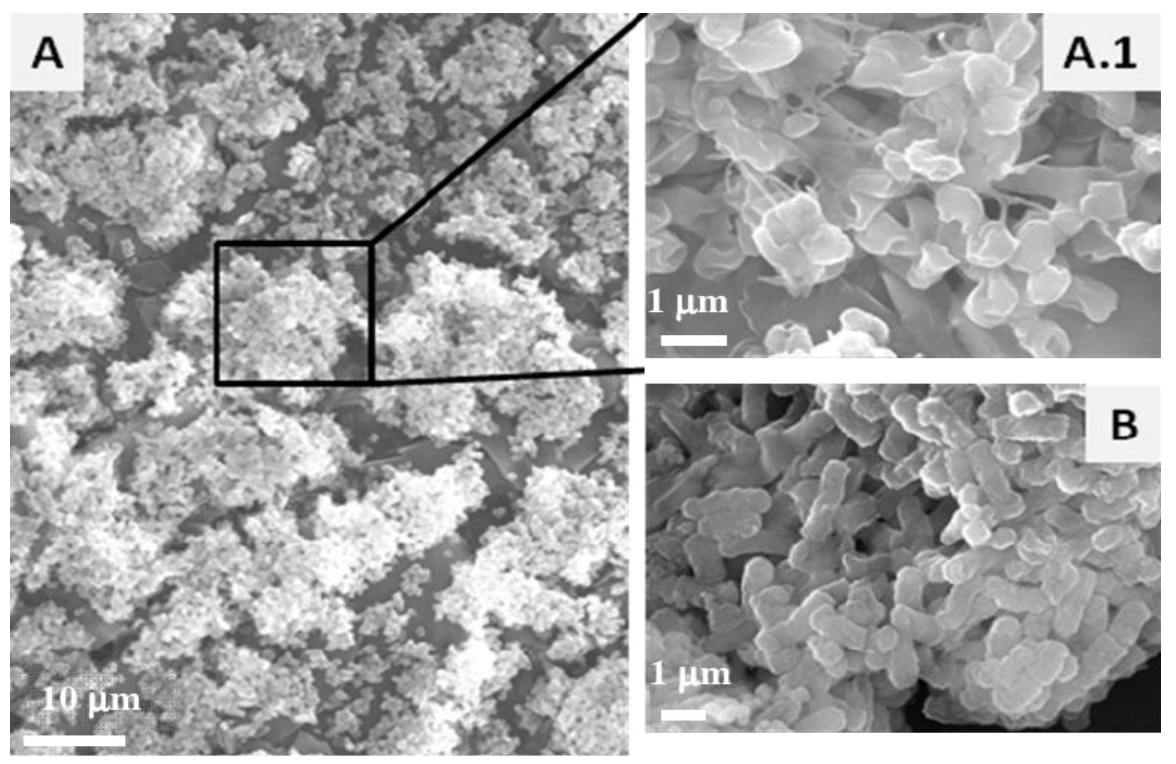

Figure 1. Bacteria structure: Planktonic versus biofilm. A panoramic view of a $12 \mathrm{~h}$ old biofilm of K.pneumoniae 703;O:1 obtained by scanning electron microscopy is shown in figure 1.A. The structural differences between bacteria organized in biofilm (1.A.1) and planktonic bacteria (1.B) were highlighted by this technique.
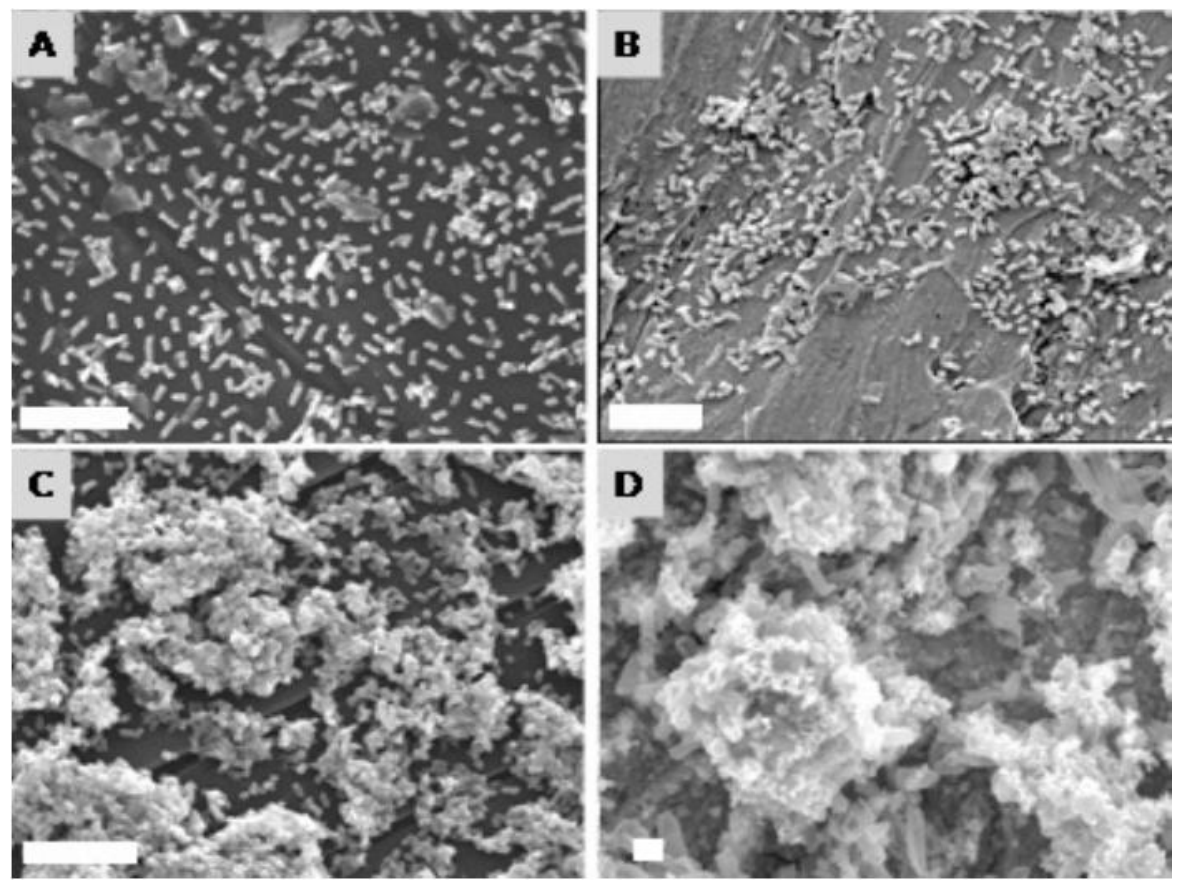

Figure 2. Bacterial biofilm assemble on different abiotic surfaces present in healthcare units. Twelve hours old biofilms assembled either on silicon (mimicking medical instruments coating e.g. catheter) or metal (mimicking water system pipes) of two different strains of $K$. pneumoniae are shown. The ability of $K$. pneumoniae 2948 to assemble biofilm either on silicon (A) or on metal (B) is reduced in comparison to K.pneumoniae 703;O:1 for both materials. The images were acquired by SEM (bars $=1 \mu \mathrm{m})$. 